The Journal of Animal \& Plant Sciences, 31(1): 2021, Page: 143-155

ISSN (print): 1018-7081; ISSN (online): 2309-8694

\title{
GENETIC VARIABILITY AND SEASONAL INFLUENCE OF FALL ARMYWORM (SPODOPTERA FRUGIPERDA (J. E. SMITH)) DAMAGE TO EARLY VEGETATIVE GROWTH OF AFRICAN MAIZE ACCESSIONS IN THE HUMID TROPICS OF SOUTH- EASTERN NIGERIA
}

\author{
E. Obok ${ }^{*}$, M. Ittah, I. Udo, E. Ojubi, J. Ulafor and S. Umoetok \\ Crop Improvement and Protection Unit, Department of Crop Science, Faculty of Agriculture, Forestry and Wildlife \\ Resources Management, University of Calabar, Calabar, Cross River State, Nigeria \\ "Corresponding author's email: e.e.obok@unical.edu.ng
}

\begin{abstract}
Fall armyworm (Spodoptera frugiperda (J. E. Smith)) (FAW) is a petrifying invasive pest of maize (Zea mays L.) in Africa. Ten tropical maize accessions from the International Plant Genetic Resources Institute (IPGRI) collections and a check (Ikom White variety) were studied, under rainfed conditions, in a multi-environment trial (MET) to assess their responses to natural infestation of FAW. The study was conducted in the 2018 first and second maize cropping seasons in Cross River State, Nigeria using a genotype $\mathrm{x}$ season interaction (GSI) model. The combined analyses of variance showed non-significant difference $(p>0.05)$ for genotypes and significant $(p \leq 0.05)$ genotype $x$ season interactions for seedling emergence, plant height, number of leaves, leaf area and FAW infestation. The most FAW-damaged maize accession in this study was TZm-304 while TZm-1427 was the least FAW-damaged maize accession. Overall, FAW infestation was significantly $(\mathrm{p} \leq 0.01)$ higher in the second maize cropping season (Akpabuyo) than in first maize cropping season (Calabar). This subsequently led to corresponding decreases in early vegetative growth in the second maize cropping season. The IPGRI African maize accessions evaluated in this study were all susceptible to FAW damage at their early vegetative growth stages. However, the infestation dynamics of FAW on maize in Africa is presently being understudied. Thus, there is a dire need for the adoption of a genotype $\mathrm{x}$ location $\mathrm{x}$ year (GLY) model in subsequent MET studies on FAW across all maize-growing States in Nigeria and selection for maize genotypes in breeding for resistance to FAW should consider early vegetative growth stage and initial establishments in the field as useful aids to selection.
\end{abstract}

Key words: Fall armyworm, Ikom White, IPGRI, maize, multi-environment trial, Nigeria.

https://doi.org/10.36899/JAPS.2021.1.0202

Published online August 26, 2020

\section{INTRODUCTION}

Fall armyworm Spodoptera frugiperda (J. E. Smith) (FAW), a noctuid moth, is a peril to the production of maize (Zea mays L.) in temperate and tropical regions of the world. There is a dire need of longterm solutions and remediations to this deleterious invasive pest, which has presently affected more than 80 different crops including grasses and legumes (Food and Agriculture Organization of the United Nations, 2018; Nagoshi et al., 2018; Prasanna et al., 2018; Toepfer et al., 2018). Though fall armyworm has its origin in the tropical regions of the Western hemisphere, it has now become a hostile pest of significant economic importance in African and Asian countries (Brévault et al., 2018; Chen et al., 2019; Kalleshwaraswamy et al., 2019; Nagoshi et al., 2017; Nagoshi et al., 2018; Otim et al., 2018). It was first reported in Nigeria in 2016 (Goergen et al., 2016). Plants of the grass family are predominant hosts of fall armyworm; maize been one of the most affected cereal.
Maize is a staple in Central and West African countries; providing food and source of income to smalland large-scale farmer in these areas. However, there have been concerns of low soil fertility, drought and biotic stress such as pest and diseases. The arrival of the fall armyworm, originally from the tropical regions of the Americas, tends to increase these present concerns. There is presently no single effective management strategy to FAW. Farmers in Africa are losing their crops to its attack in quite an alarming rate. In Africa, maize production loss estimates caused as a result of FAW infestations ranges from $50 \%$ to $100 \%$ (Baudron et al., 2019; Rwomushana et al., 2018).

Though there have been reports on the use of $B t$ technology (Burtet et al., 2017; Oliveira et al., 2018), biological (Hay-Roe et al., 2016; Meagher et al., 2016) and chemical (Burtet et al., 2017; Food and Agriculture Organization of the United Nations, 2018) control measures for FAW, these reports have failed to provide the necessary solution needed to match the geometric crop losses accompanying fall armyworm infestations. Maize crops are attacked by fall armyworm at larval 
stages, the most destructive phase in armyworm lifecycle. However, the adult (males and females) are not destructive to host crops. The attack is mostly on the young leaves. Though the larvae could also feed on maize silk and tassel tissues, the ultimate feeding site preference is the succulent and tender leaf tissues, especially at whorl stages. Apparently, the mature leaves are classified as unsuitable site for the growth and development of fall armyworm (Pannuti et al., 2016). The distinct characteristic mouthpart of fall armyworm larvae encourages rapid defoliation which often increases with the age of the larvae. The first to third larval instar causes injuries to maize plant leaves ranging from perforations to defoliations. Maize leaf stalks and ribs are less likely affected by fall armyworm. Meanwhile, the critical period for early vegetative growth of maize (i.e. establishment in the field) is usually between the first four to six weeks. This growth period coincides with the early period of FAW incidence and infestation and could lead up to a $20 \%$ yield reduction at a larvae-per-plant density of 0.2 to 0.8 (Marenco et al., 1992). Since the first few weeks of maize establishment in the field overlaps with the early damage to the crop by FAW, especially the vegetative growth stages, there could be a huge threat capable of destroying the entire growth and yield potentials of the plants.

Aside from the biotic pressure of FAW on the growth of maize, seasonal variations could also have influence on incidence and infestation levels of FAW on the maize crop. In the South Eastern humid tropics of Nigeria, there are basically two major cropping seasons for maize production. While the first (early) cropping season usually commences in mid-March, the second (late) cropping season starts in mid-September in Nigeria. To the best of our knowledge and available published literature, this study is the first to look at possible influences that the two main growing seasons of maize could have on the level of FAW infestation on maize grown in the humid tropics of Nigeria. Notably, present reports have only highlighted either the presence or absence of FAW on maize and other related host crops without giving information on seasonal variations. The present study was designed to examine the response of different African maize accessions to FAW damage under natural infestation in the humid tropical agroecology of South Eastern Nigeria during early and late maize cropping seasons. The variance components of the early vegetative growth traits were also estimated. The collection of information is paramount, especially for an invasive pest of such economic magnitude. From the depth of results obtained from the present study, we are confident that new vistas for research will arouse interest on in-depth studies on FAW damage in maize across different maize-growing areas and environments in Nigeria.

\section{MATERIALS AND METHODS}

Ten tropical maize accessions of diverse African origins were sourced through International Institute of Tropical Agriculture (IITA), Ibadan, Nigeria, from Biodiversity International (formerly International Plant Genetic Resources Institute), Italy (Table 1). Complete descriptors of each of the maize accessions are available at http://my.iita.org/accession2/ under the heading 'Mazie collection'. Ikom White, a white floury maize variety bred for South Eastern Nigeria (Eijnatten, 1965), was used as a 'check'. The experiments were conducted in two seasons in 2018 namely, the Cross River State Agricultural Development Programme experimental and demonstration farms in Calabar (N04 ${ }^{\circ} 57^{\prime}$ E08 $20^{\prime}$ ' at an elevation of $35 \mathrm{~m}$ above sea level) (i.e. first cropping season) and Akpabuyo ( ${\mathrm{N} 04^{\circ}}^{\circ} 56^{\prime}$ E08 ${ }^{\circ} 23^{\prime}$ at an elevation of $22 \mathrm{~m}$ above sea level) (i.e. second cropping season).

Table 1. Documented origins of African maize accessions and 'check' variety used in the present study.

\begin{tabular}{lll}
\hline Genotype & Reported African Origin & Source \\
\hline Accession & & \\
TZm-109 & Republic of Benin & Biodiversity International \\
TZm-299 & Republic of Chad & Biodiversity International \\
TZm-304 & Republic of Chad & Biodiversity International \\
TZm-378 & Republic of Congo & Biodiversity International \\
TZm-687 & Ghana & Biodiversity International \\
TZm-1097 & Unconfirmed & Biodiversity International \\
TZm-1153 & Unconfirmed & Biodiversity International \\
TZm-1163 & Burkina Faso & Biodiversity International \\
TZm-1241 & Unconfirmed & Biodiversity International \\
TZm-1427 & Republic of Congo & Biodiversity International \\
Check & & \\
Ikom White variety & Nigeria & Cross River State Agricultural Development Programme \\
\hline
\end{tabular}


The experiments were both carried out on a 12month fallow farmland which were not previously planted to either maize crop or crops documented as hosts to FAW. Six representative soil samples were randomly collected with an ungalvanized soil auger from each of the experimental site, at a depth of $15 \mathrm{~cm}$. The samples were bulked according to each block (replicate) for soil physical and chemical properties analyses at the Department of Soil Science Analytical Laboratory, University of Calabar, Calabar. Student t-tests were conducted for soil properties and weather data obtained from Calabar and Akpabuyo. In the laboratory, wet towel germination test was conducted to ascertain the viability of the maize seeds. A 100-percent seed viability was recorded for all the maize genotypes, allowing the planting of one seed per hole in the field. The first experiment began in the field on 15 March 2018 (i.e. the first cropping season) while the second experiment commenced on 15 September 2018 (i.e. the second cropping season). Both experiments were conducted under rainfed conditions. In each of the seasons, the experiment was laid out in a randomized complete block design (RCBD) with three replications (i.e. blocks) and each block had eleven (11) treatment plots representing 11 maize genotypes (i.e. ten tropical maize accessions and one check variety). The plant spacing was $75 \mathrm{~cm} \mathrm{x}$ $25 \mathrm{~cm}$ at a seeding depth of $2-3 \mathrm{~cm}$.

Seedling emergence was observed at seven days after sowing (DAS) and expressed as a percentage of the total number of plants emerged (i.e. visible true leaves above soil level) to the total number of seeds sown in each treatment plot. For analysis of variance (ANOVA), data on seedling emergence count was transformed as $[\log ($ counts +1$)]$ to reduce the heterogeneity of variance for seedling emergence. Each treatment plot size measured $3 \mathrm{~m} \times 1.5 \mathrm{~m}$, giving a total number of 30 plants per treatment plot i.e. 53,333 plants/ha. Inorganic fertilizer, NPK 15:15:15, was applied at the rate of $400 \mathrm{~kg} / \mathrm{ha}$ by ring method at sowing (i.e. $60 \mathrm{kgN} / \mathrm{ha}$ ). Postemergence weed control (hand hoeing) was carried out at 14 DAS. In both seasons, fall armyworm incidence occurred as natural infestations. There were no FAW management practices carried out i.e. no chemical, biological and/or cultural control measure was applied in the fields. Scouting and identification of FAW infestation started at 3-7 days after seedling emergence. All the plants, especially the leaves, in each of the treatment plots were examined twice daily - in the morning (07:30) and evening (16:00). At 14 DAS and 28 DAS, the number of plants naturally infested by fall armyworm were recorded and expressed as a percentage of the total number of plants per treatment plot. Data on selected growth traits at the early vegetative stages of the maize plants were collected at 14 DAS and 28 DAS. These included plant height $(\mathrm{PH})(\mathrm{cm})$, stem width $(\mathrm{SW})(\mathrm{mm})$, average number of leaves (NL) per plant, leaf area (LA) $\left(\mathrm{cm}^{2}\right)$ and leaf area index (LAI) (Elings, 2000).

Combined ANOVA using GenStat 16.1 (VSN International Ltd., Hemel Hempstead, England, United Kingdom), were conducted for genotypes over season to estimate the mean performance and response of the maize genotypes to early vegetative growth and fall armyworm infestation, respectively. Significant mean differences were compared using Duncan's multiple range test (DMRT) (Gomez and Gomez, 1984). Variance components and expected mean squares for genotypes and genotype $\mathrm{x}$ season interactions were estimated (Kehr and Gardner, 1960; Yassin, 2009). Genotypic coefficient of variability (GCV) and phenotypic coefficient of variability (PCV) were also calculated (Falconer, 1960). The model used for the analysis was the genotype $\mathrm{x}$ season interaction (GSI) model adopted from Can and Yoshida (1999). Here, each season was considered as a test environment. Thus, ANOVA of the RCBD model which assumed independent and heterogeneous error variance of seasons was carried out to assess mean differences.

$$
\mathbf{Y}_{\mathbf{i j k l}}=\mathbf{M}+\mathbf{S}_{\mathbf{i}}+\mathbf{R}(\mathbf{S})_{\mathbf{j}(\mathbf{i})}+\mathbf{G}_{\mathbf{k}}+\mathbf{G S}_{\mathbf{i k}}+\mathbf{e}_{\mathbf{i j k l}}
$$

Where: $\mathrm{Y}_{i j k l}=$ the measurement on plot $l$ in season $i$, block $j$, containing genotype $k ; \mathrm{M}=$ the overall mean of all plots in all seasons; $\mathrm{S} i=$ the effect of season $i$; $(\mathrm{S})_{j(i)}=$ the effect of replicate $\mathrm{j}$ within season $\mathrm{i} ; \mathrm{G}_{k}=$ the effect of genotype $k$; $\mathrm{GS}_{i k}=$ the interaction of genotype $i$ with season $\mathrm{k}$ and eijkl = the plot residual. The analysis of variance for the combined data for the two cropping seasons was as follows, assuming all factors random:

Table 2. Combined analysis of variance for early vegetaive growth and FAW infestation.

\begin{tabular}{lcll}
\hline Source of Variation & df & MS & Expected MS \\
\hline Season (S) & $\mathrm{s}-1$ & $\mathrm{MS}$ & $\sigma_{e}^{2}+r \sigma_{g s}^{2}+g \sigma_{r(s)}^{2}+r g \sigma_{s}^{2}$ \\
Replicates within season & $\mathrm{s}(\mathrm{r}-1)$ & $\mathrm{MS}_{\mathrm{Rep}(\mathrm{S})}$ & $\sigma_{e}^{2}+g \sigma_{r(s)}^{2}$ \\
Genotype $(\mathrm{G})$ & $\mathrm{g}-1$ & $\mathrm{MS}_{\mathrm{G}}$ & $\sigma_{e}^{2}+r \sigma_{g s}^{2}+s r \sigma_{g}^{2}$ \\
$\mathrm{G} x \mathrm{~S}$ interaction & $(\mathrm{s}-1)(\mathrm{g}-1)$ & $\mathrm{MS}_{\mathrm{GS}}$ & $\sigma_{e}^{2}+r \sigma_{g s}^{2}$ \\
Pooled error $(\mathrm{E})$ & $\mathrm{s}(\mathrm{r}-1)(\mathrm{g}-1)$ & $\mathrm{MS}$ & $\sigma_{e}^{2}$ \\
\hline $\mathrm{s}=$ season, $\mathrm{g}=$ genotype, $\mathrm{r}=$ replicate, $\sigma_{g}^{2}=$ genotypic variance, $\sigma_{s}^{2}=$ season variance, $\sigma_{g s}^{2}=$ genotype x season interaction variance, \\
$\sigma_{r(s)}^{2}=$ replicate within season variance, $\sigma_{e}^{2}=$ pooled error variance, df = degree of freedom, MS = Mean square.
\end{tabular}




\section{RESULTS AND DISCUSSION}

Soil properties and weather conditions in the first and second maize cropping seasons: Analytical test results of physical and chemical soil properties at Calabar and Akpabuyo showed that these soils were loamy sand in texture with low total nitrogen $(0.11-0.14 \%)$ and slightly acidic to near neutral in $\mathrm{pH}(5.2-6.9)$. Akpabuyo soil was richer in available soil phosphorus and potassium ion whereas Calabar soil had higher organic carbon content which was, however, not significantly different $(\mathrm{p}>0.05)$ from Akpabuyo soil. Although lacking in $\mathrm{Al}^{3+}$, exchangeable cations and effective cation exchange capacity were generally higher in Akpabuyo soils than in Calabar soils, ranging from 1.49 to $7.20 \mathrm{cmol} \mathrm{kg}^{-1}$. It was not unusual to record a higher base saturation in Akpabuyo than in Calabar given the high exchangeable acidity and low exchangeable bases obtained in this area (Akpan-Idiok, 2012). These observations were in consonant with soil analysis reports for south-eastern soils of Nigeria (Akpan-Idiok, 2012; Onwudike, 2015). The weather conditions (WorldWeatherOnline, 2018) were characteristically marked by high rainfall in the second cropping seasons (mid-September). Overall, these often have implications for high soil erosion and leaching of soil nutrients, poor fertility status as primary features peculiar to most soils of the humid tropical rainforest of south-eastern Nigeria (Akpan-Idiok, 2012). Meanwhile, at the commencement of the early season planting, rainfall (and rainy days) were lower as the rains were yet to be fully established by mid-March. The UV index was high, ranging from 6 to 7 with an accompanying sun hour (sun days) ranging from 155 to $254 \mathrm{hr}$, respectively. Overall, Akpabuyo and Calabar were not significantly different $(p>0.05)$ in terms of UV index, temperature and wind during the study. The average temperature, relative humidity and air pressure were comparatively low in the first cropping season during the study. These results were in consonant with recent yearly weather reports for Akpabuyo and Calabar (Nigerian Meteorological Agency (NiMET), 2018).

Early vegetative growth and influence of FAW on African maize accessions: Seasonal analysis of variance for the early vegetative growth traits and response to FAW damage (Figures 1, 2 and 3) of all the maize genotypes at Calabar and Akpabuyo showed significant differences $(p \leq)$. The highest percentage of seedlings emergence at 7DAS was recorded by accession TZm$1163(98.15 \pm 1.85 \%)$ in the second planting season, at Akpabuyo. Conversely, TZm-1163 had the least number of seedlings that emerged $(33.33 \pm 3.2 \%)$ at 7 DAS sowing in the first planting season, at Calabar. In both seasons, the check variety, Ikom White, had an average seedling emergence ranging from $60 \%$ to $80 \%$ at $7 \mathrm{DAS}$. In the first cropping season, plant height ranged from $13.15 \mathrm{~cm}$ (TZm-1163) to $30.8 \mathrm{~cm}$ (Ikom White) at 2WAS. Though average plant height in the second cropping season ranged from $8.5 \mathrm{~cm}$ (TZm-1427) to $12.3 \mathrm{~cm}$ (TZm-1153) at $2 \mathrm{WAS}$, the maize genotypes were not significantly different $(\mathrm{p}>0.05)$ from the check in height $(8.9 \pm 0.2$ $\mathrm{cm})$. However, the check variety at $2 \mathrm{WAS}$ was not significantly different $(\mathrm{p}>0.05)$ from TZm-109, TZm299 and TZm-304 in the first cropping season. At 4WAS, in both seasons, TZm-304 was significantly $(\mathrm{p} \leq 0.05)$ taller than all the maize genotypes. Overall, the maize genotypes were taller at both $2 \mathrm{WAS}$ and $4 \mathrm{WAS}$ in the first planting season (Calabar) than in the second planting season (Akpabuyo). The average stem width at $2 \mathrm{WAS}$ ranged from $10.0 \pm 0.6 \mathrm{~mm}$ (TZm-1241 at Calabar) to $18.3 \pm 1.2 \mathrm{~mm}$ (TZm-304 at Calabar); these observations were not significantly different $(\mathrm{p}>0.05)$ from Ikom White at Akpabuyo and Calabar, respectively. TZm-1241 had the thickest stems $(19.3 \pm 0.3 \mathrm{~mm})$ in Akpabuyo and the thinnest stems $(12.1 \pm 1.0 \mathrm{~mm})$ in Calabar at 4 WAS. Meanwhile, though the accessions differed in stem width, the checks had similar stem widths $(16 \mathrm{~mm})$ and were not significantly different $(p>0.05)$ from all the accessions at Calabar and Akpabuyo. The highest number of leaves (6.2 \pm 0.4$)$ were obtained in Akpabuyo (TZm-1163) at 2WAS. TZm-1163 also recorded the lowest number of leaves $(4.2 \pm 0.2)$ in Calabar at 2 WAP. Ikom White had significantly $(\mathrm{p} \leq 0.05)$ lower number of leaves $(5.0 \pm 0.2)$ when compared with TZm-1163 (6.2 \pm 0.4$)$ in Akpabuyo at 2WAS. In Calabar, Ikom White was significantly different $(\mathrm{p} \leq 0.05)$ from TZm-1163, TZm-1097 and TZm-1241 in the number of leaves recorded at 2WAS, but at $4 \mathrm{WAS}$ in Calabar, the check had the highest number of leaves $(8.1 \pm 0.4)$ and was statistically similar $(p>0.05)$ to other accessions. Remarkably, the number of leaves had reduced for accessions TZm-109, TZm1097, TZm-1163, TZm-1241, TZm-1427, TZm-299, TZm-378 and Ikom White at 4WAS. Accession TZm-304 had the largest leaf area at 2WAS $\left(505.4 \pm 68.5 \mathrm{~cm}^{2}\right)$ and 4WAS $\left(1348.1 \pm 201 \mathrm{~cm}^{2}\right)$ in Akpabuyo. Comparatively, across seasons, larger leaf area was observed for all the accessions in the second cropping season, except for Ikom White which also had a lower leaf area $(237.5 \pm 56.3$ $\left.\mathrm{cm}^{2}\right)$ in Akpabuyo than in Calabar $\left(266.8 \pm 46.1 \mathrm{~cm}^{2}\right)$ at $2 \mathrm{WAS}$ and vice versa at $4 \mathrm{WAS}$.

TZm-1097 had the lowest leaf area at 2WAS during the first cropping season whereas in the second cropping season TZm-1241 recorded the least leaf area. At 4WAS, all the accessions were not significantly different $(p>0.05)$ from the check in terms of leaf area observations at Calabar. A similar trend was observed for the check at Akpabuyo. The leaf area index (LAI) of the maize genotypes at 2 WAS ranged from 0.25 to 0.65 in Calabar and 0.55 to 1.54 at Akpabuyo. Overall, the LAI was higher in the second cropping season at 4WAS. Ikom White showed the highest LAI $(1.48 \pm 0.2)$ in Calabar and 
was not significantly different $(\mathrm{p}>0.05)$ from TZm-1163 $(0.52 \pm 0.1)$ and other maize accessions at $4 \mathrm{WAS}$. Although TZm-1427 had the lowest LAI $(1.76 \pm 0.3)$ at 4WAS in Akpabuyo, this was not significantly different $(p>0.05)$ from the check (1.84 \pm 0.8$)$. Other accessions in Akpabuyo had LAI values that were higher than Ikom White and TZm-1427. When FAW infestation comparisons were made between seasons it was observed that the mean severity of damage on the maize accessions were generally higher in the second planting season (Akpabuyo) than in the first planting season (Calabar) (Table 4), with exception to Ikom White which recorded low FAW infestation at 2WAS and 4WAS at Akpabuyo. From the combined ANOVA, TZm-299 recorded the highest seedling emergence $(88.89 \%)$ while the lowest percentage of seedlings that emerged at 7DAS was recorded for TZm-1097 (61.11\%) which was not significantly different $(\mathrm{p}>0.05)$ from the check $(69.44 \%)$ and other accessions except TZm-299, TZm-304 and TZm-687. The tallest plants at 2WAS were Ikom White $(19.9 \mathrm{~cm})$ and were significantly different $(\mathrm{p} \leq 0.05)$ from accessions TZm-378 $(13.1 \mathrm{~cm})$, TZm-1241 $(13.3 \mathrm{~cm})$ and TZm-1163 (12.5 cm). At 4WAS TZm-304 was taller $(39.5 \mathrm{~cm})$ than Ikom White $(29.1 \mathrm{~cm})$ but not significantly different $(\mathrm{p}>0.05)$ from other accessions. The average stem girth ranged from $11.2 \mathrm{~mm}$ to $17.5 \mathrm{~mm}$ at $2 \mathrm{WAS}$. Ikom White recorded $12.5 \mathrm{~mm}$ and was not significantly different $(\mathrm{p}>0.05)$ from other maize accessions except TZm-304 ( $\mathrm{p} \leq 0.05)$. When the plants grew to 4WAS, the trend in stem width differences was similar for the thickest and thinnest stems, TZm-304 and TZm-1163 accessions, respectively. Combined ANOVA did not show a significant difference $(p>0.05)$ for number of leaves per plant and leaf area index (LAI) at $4 \mathrm{WAS}$ (Table 5).

Table 3. Soil properties and weather conditions at Calabar and Akpabuyo in the 2018 maize cropping seasons.

\begin{tabular}{|c|c|c|c|}
\hline Soil Properties ${ }^{\dagger}$ & $\begin{array}{l}\text { Early Season }{ }^{1} \\
\text { (Calabar) }\end{array}$ & $\begin{array}{l}\text { Late Season }^{2} \\
\text { (Akpabuyo) }\end{array}$ & $\begin{array}{l}\text { t-test } \\
(\mathrm{df}=10)\end{array}$ \\
\hline \multicolumn{4}{|l|}{ Texture $\left(\mathrm{g} \mathrm{kg}^{-1}\right)$} \\
\hline Sand & $800 \pm 3.3$ & $839 \pm 3.3$ & $* * *$ \\
\hline Silt & $123 \pm 3.0$ & $47 \pm 3.1$ & $* * *$ \\
\hline Clay & $77 \pm 0.5$ & $114 \pm 3.7$ & $* * *$ \\
\hline Textural Class (USDA) & Loamy Sand & Loamy Sand & \\
\hline $\mathrm{pH}$ (in $1: 2.5$ Soil- $\mathrm{H}_{2} \mathrm{O}$ ratio) & $5.2 \pm 0.09$ & $6.9 \pm 0.41$ & $* * *$ \\
\hline Organic Carbon $(\%)$ & $1.59 \pm 0.018$ & $1.17 \pm 0.237$ & ns \\
\hline Total Nitrogen $(\%)$ & $0.11 \pm 0.003$ & $0.14 \pm 0.006$ & ns \\
\hline Available Phosphorus $\left(\mathrm{mg} \mathrm{kg}^{-1}\right)$ & $21.16 \pm 1.020$ & $36.27 \pm 0.96$ & $* * *$ \\
\hline \multicolumn{4}{|l|}{ Exchangeable Cations $\left(\mathrm{cmol} \mathrm{kg}^{-1}\right)$} \\
\hline $\mathrm{Ca}^{2+}$ & $3.53 \pm 0.067$ & $7.20 \pm 3.160$ & $* * *$ \\
\hline $\mathrm{Mg}^{2+}$ & $1.53 \pm 0.065$ & $2.07 \pm 0.521$ & ns \\
\hline$K^{+}$ & $0.12 \pm 0.007$ & $1.62 \pm 0.128$ & $* * *$ \\
\hline $\mathrm{Na}^{+}$ & $0.06 \pm 0.003$ & $1.49 \pm 0.309$ & $* * *$ \\
\hline \multicolumn{4}{|l|}{ Exchangeable Acidity $\left(\mathrm{cmol} \mathrm{kg}^{-1}\right)$} \\
\hline $\mathrm{Al}^{3+}$ & $0.80 \pm 0.012$ & $0.00 \pm 0.00$ & $* * *$ \\
\hline$H^{+}$ & $1.13 \pm 0.067$ & $0.60 \pm 0.10$ & $* * *$ \\
\hline Effective Cation Exchange Capacity $\left(\mathrm{cmol} \mathrm{kg}^{-1}\right)$ & $7.19 \pm 0.110$ & $12.98 \pm 4.120$ & $* * *$ \\
\hline Base Saturation $(\%)$ & $72.9 \pm 0.58$ & $94.8 \pm 0.73$ & $* * *$ \\
\hline Weather Conditions ${ }^{\dagger \prime}$ & $\begin{array}{l}\text { Early Season }^{1} \\
\text { (Calabar) }\end{array}$ & $\begin{array}{l}\text { Late Season }^{2} \\
\text { (Akpabuyo) }\end{array}$ & $\begin{array}{l}\text { t-test } \\
(\mathrm{df}=10)\end{array}$ \\
\hline Rainfall (mm) & $113.04(63.06-163.01)$ & $334.46(271.92-397.00)$ & ** \\
\hline Rainy Days & $25.5(24-27)$ & $29.5(29-30)$ & * \\
\hline Relative Humidity (\%) & $77.5(77-78)$ & $85.5(83-88)$ & ** \\
\hline Ultraviolet Radiation Index & $6.5(6-7)$ & $5.5(5-6)$ & ns \\
\hline Ambient Temperature $\left({ }^{\circ} \mathrm{C}\right)$ & $26.5(26-27)$ & $27(25-29)$ & ns \\
\hline Air Pressure (mb) & $1010.9(1010.7-1011.1)$ & $1011.85(1012.2-1011.5)$ & $*$ \\
\hline Sun Hour (hr) & $247.3(234.0-253.5)$ & $155.8(155.5-156.0)$ & $* * *$ \\
\hline Sun Days & $4.5(4-5)$ & $0.5(0-1)$ & $* * *$ \\
\hline Wind (kmph) & $6.15(6.0-6.3)$ & $5.9(4.6-7.2)$ & ns \\
\hline
\end{tabular}


Table 4. Early vegetative growth and fall armyworm infestation on African maize accessions in early and late cropping seasons.

\begin{tabular}{|c|c|c|c|c|c|c|c|c|c|c|c|c|c|}
\hline \multirow[b]{2}{*}{ Genotype } & \multirow{2}{*}{$\begin{array}{l}\text { Seedling } \\
\text { Emergence (\%) }\end{array}$} & \multicolumn{2}{|c|}{ Plant Height (cm) } & \multicolumn{2}{|c|}{ Stem Width (mm) } & \multicolumn{2}{|c|}{ Number of Leaves } & \multicolumn{2}{|c|}{ Leaf Area $\left(\mathrm{cm}^{2}\right)$} & \multicolumn{2}{|c|}{ Leaf Area Index } & \multicolumn{2}{|c|}{$\begin{array}{l}\text { Fall Armyworm } \\
\text { Infestation (\%) }\end{array}$} \\
\hline & & 2WAS & 4WAS & 2WAS & 4WAS & 2WAS & 4WAS & 2WAS & 4WAS & 2WAS & 4WAS & 2WAS & 4WAS \\
\hline \multicolumn{14}{|l|}{$\begin{array}{l}\text { Early Season } \\
\text { (Calabar) }\end{array}$} \\
\hline TZm-109 & $77.78 \pm 3.21^{\text {abcdefg }}$ & $24.4 \pm 6.7^{\mathrm{abc}}$ & $32.6 \pm 9.9^{\text {bcdef }}$ & $11.7 \pm 2.8^{\mathrm{bc}}$ & $12.8 \pm 2.9^{\mathrm{de}}$ & $5.6 \pm 0.5^{\mathrm{abcd}}$ & $7.3 \pm 1.3^{\mathrm{abc}}$ & $153.2 \pm 92.5^{\text {cde }}$ & $194.4 \pm 80.1^{\mathrm{d}}$ & $0.62 \pm 0.5^{\mathrm{bc}}$ & $0.85 \pm 0.5^{\mathrm{de}}$ & $4.17 \pm 2.1^{\text {ef }}$ & $16.67 \pm 2.1^{\mathrm{d}}$ \\
\hline TZm-1097 & $51.85 \pm 14.46^{\mathrm{hij}}$ & $17.9 \pm 3.0^{\text {cdefg }}$ & $44.1 \pm 6.4^{\mathrm{abc}}$ & $11.7 \pm 1.4^{\mathrm{bc}}$ & $13.5 \pm 1.4^{\text {cde }}$ & $4.3 \pm 0.3^{\text {fg }}$ & $6.7 \pm 0.7^{7 \mathrm{abcd}}$ & $50.9 \pm 22.6^{\mathrm{e}}$ & $150.4 \pm 31.4^{\mathrm{d}}$ & $0.60 \pm 0.2^{\mathrm{bc}}$ & $0.56 \pm 0.2^{\mathrm{e}}$ & $10.42 \pm 5.5^{\text {cdef }}$ & $25.00 \pm 10.8^{\mathrm{cd}}$ \\
\hline $\mathrm{TZm}-1153$ & $68.52 \pm 4.90^{\text {cdefgh }}$ & $20.8 \pm 5.0^{\text {bcde }}$ & $46.3 \pm 14.1^{\mathrm{ab}}$ & $13.0 \pm 2.5^{\mathrm{abc}}$ & $14.6 \pm 1.8^{\text {abcde }}$ & $4.5 \pm 0.4^{\mathrm{efg}}$ & $7.9 \pm 0.7^{\mathrm{a}}$ & $114.5 \pm 39.9^{\text {cde }}$ & $170.1 \pm 41.7^{\mathrm{d}}$ & $0.44 \pm 0.3^{\mathrm{bc}}$ & $0.72 \pm 0.2^{\mathrm{de}}$ & $6.25 \pm 6.3^{\text {def }}$ & $20.83 \pm 5.5^{\mathrm{cd}}$ \\
\hline TZm-1163 & $33.33 \pm 3.2^{\mathrm{j}}$ & $13.5 \pm 1.5^{\text {defgh }}$ & $27.8 \pm 5.8^{\text {bcdef }}$ & $11.0 \pm 0.9^{\mathrm{bc}}$ & $12.9 \pm 1.0^{\mathrm{de}}$ & $4.2 \pm 0.2^{\mathrm{g}}$ & $6.8 \pm 0.3^{3 \mathrm{abcd}}$ & $86.0 \pm 47.5^{\mathrm{de}}$ & $144.8 \pm 34.7^{\mathrm{d}}$ & $0.34 \pm 0.1^{c}$ & $0.52 \pm 0.1^{\mathrm{e}}$ & $2.08 \pm 2.1^{\mathrm{f}}$ & $20.83 \pm 4.2^{\text {cd }}$ \\
\hline $\mathrm{TZm}-1241$ & $74.07 \pm 4.90^{\text {bcdefgh }}$ & $17.4 \pm 3.4^{\text {cdefgh }}$ & $34.5 \pm 3.8^{\text {abcde }}$ & $10.0 \pm 0.6^{c}$ & $12.1 \pm 1.0^{\mathrm{e}}$ & $4.3 \pm 0.3^{\text {fg }}$ & $6.7 \pm 0.6^{\text {abcd }}$ & $150.2 \pm 47.4^{\text {cde }}$ & $190.3 \pm 36.4^{\mathrm{d}}$ & $0.25 \pm 0.2^{\mathrm{c}}$ & $0.70 \pm 0.2^{\mathrm{de}}$ & $6.25 \pm 0.0^{\mathrm{def}}$ & $16.67 \pm 2.1^{\mathrm{d}}$ \\
\hline TZm-1427 & $83.33 \pm 3.31$ abcdef & $19.8 \pm 3.3^{\text {bcdef }}$ & $37.4 \pm 3.5^{\mathrm{abcd}}$ & $15.3 \pm 2.2^{\mathrm{abc}}$ & $16.2 \pm 2.0^{\text {abcde }}$ & $4.9 \pm 0.3^{\text {cdefg }}$ & $6.8 \pm 0.7^{\mathrm{abcd}}$ & $147.3 \pm 63.7^{\text {cde }}$ & $195.9 \pm 51.6^{\mathrm{d}}$ & $0.45 \pm 0.2^{\mathrm{bc}}$ & $0.74 \pm 0.3^{\mathrm{de}}$ & $12.50 \pm 12.5^{\text {bcdef }}$ & $33.33 \pm 13.7^{\mathrm{bcd}}$ \\
\hline TZm-299 & $90.74 \pm 4.90^{\mathrm{abc}}$ & $26.7 \pm 2.3^{\mathrm{ab}}$ & $43.6 \pm 8.9^{\mathrm{abc}}$ & $15.4 \pm 1.4^{\mathrm{abc}}$ & $16.2 \pm 1.5^{\text {abcde }}$ & $5.0 \pm 0.3^{\text {bcdefg }}$ & $7.9 \pm 0.3^{\mathrm{a}}$ & $199.5 \pm 35.0^{\text {cde }}$ & $246.8 \pm 11.5^{\mathrm{cd}}$ & $0.45 \pm 0.2^{\mathrm{bc}}$ & $1.04 \pm 0.1^{\mathrm{de}}$ & $14.58 \pm 9.1^{\text {bcdef }}$ & $31.25 \pm 3.6^{\mathrm{bcd}}$ \\
\hline TZm-304 & $94.44 \pm 1.10^{\mathrm{ab}}$ & $26.8 \pm 2.0^{\mathrm{ab}}$ & $51.1 \pm 5.7^{\mathrm{a}}$ & $18.3 \pm 1.2^{\mathrm{a}}$ & $18.7 \pm 1.2^{\mathrm{ab}}$ & $5.4 \pm 0.2^{\text {abcde }}$ & $8.0 \pm 0.4^{\mathrm{a}}$ & $171.7 \pm 70.3^{\text {cde }}$ & $259.6 \pm 43.8^{\mathrm{cd}}$ & $0.45 \pm 0.2^{\mathrm{bc}}$ & $1.12 \pm 0.2^{\text {cde }}$ & $47.92 \pm 5.5^{\mathrm{a}}$ & $70.83 \pm 5.5^{\mathrm{a}}$ \\
\hline $\mathrm{TZm}-378$ & $79.63 \pm 9.26^{\text {abcdefg }}$ & $16.7 \pm 4.2^{\text {cdefgh }}$ & $27.8 \pm 5.1^{\text {bcdef }}$ & $13.3 \pm 2.7^{\mathrm{abc}}$ & $14.4 \pm 2.3^{\text {bcde }}$ & $4.8 \pm 0.2^{\text {cdefg }}$ & $7.6 \pm 1.2^{\mathrm{ab}}$ & $90.0 \pm 56.2^{\mathrm{de}}$ & $161.4 \pm 47.9^{\mathrm{d}}$ & $0.39 \pm 0.2^{\mathrm{c}}$ & $0.71 \pm 0.3^{\mathrm{de}}$ & $41.67 \pm 14.6^{\mathrm{abc}}$ & $58.33 \pm 17.1^{\mathrm{ab}}$ \\
\hline $\mathrm{TZm}-687$ & $88.89 \pm 3.21^{\mathrm{abcd}}$ & $21.2 \pm 1.8^{\mathrm{bcd}}$ & $35.9 \pm 6.1^{\text {abcde }}$ & $15.5 \pm 2.9^{\mathrm{abc}}$ & $16.7 \pm 2.6^{\text {abcde }}$ & $4.7 \pm 0.1^{\text {defg }}$ & $7.6 \pm .06^{\mathrm{ab}}$ & $83.7 \pm 16.0^{\text {de }}$ & $138.2 \pm 22.7^{\mathrm{d}}$ & $0.41 \pm 0.2^{\mathrm{bc}}$ & $0.56 \pm 0.1^{\mathrm{e}}$ & $20.83 \pm 9.1^{\text {abcdef }}$ & $35.42 \pm 9.1 \mathrm{bcd}$ \\
\hline Ikom White & $79.63 \pm 6.68^{\text {abcdefg }}$ & $30.8 \pm 2.73^{\mathrm{a}}$ & $43.7 \pm 2.5^{\mathrm{abc}}$ & $14.5 \pm 2.0^{\mathrm{abc}}$ & $16.2 \pm 1.1^{\text {abcde }}$ & $5.3 \pm 0.1^{\text {abcde }}$ & $8.1 \pm 0.4^{\mathrm{a}}$ & $266.8 \pm 46.1^{\mathrm{cd}}$ & $342.2 \pm 26.5^{\mathrm{cd}}$ & $0.65 \pm 0.3^{\text {bc }}$ & $1.48 \pm 0.2^{\text {bcde }}$ & $25.00 \pm 12.5^{\text {abcdef }}$ & 43.75 $\pm 12.5^{\text {abcd }}$ \\
\hline \multicolumn{14}{|l|}{$\begin{array}{l}\text { Late Season } \\
\text { (Akpabuyo) }\end{array}$} \\
\hline $\mathrm{TZm}-109$ & $5.74 \pm 3.34^{\mathrm{efgh}}$ & $.7 \pm 1.1^{\mathrm{gh}}$ & $27.0 \pm 1.7^{\text {cdef }}$ & $16.2 \pm 2.3^{\mathrm{abc}}$ & $18.4 \pm 0.7^{\mathrm{ab}}$ & $5.5 \pm 0.4^{\text {abcde }}$ & $5.3 \pm 0.9^{\mathrm{d}}$ & $498.9 \pm 117.0^{\mathrm{a}}$ & $1056.0 \pm 179.0^{\mathrm{ab}}$ & $1.54 \pm 0.5^{\mathrm{a}}$ & $3.15 \pm 1.1^{\mathrm{ab}}$ & $41.67 \pm 11^{\mathrm{abc}}$ & $52.08 \pm 11.0^{\mathrm{abc}}$ \\
\hline TZm-1097 & $0.37 \pm 4.04^{\text {cdefgh }}$ & $0.0 \pm 1.6^{\mathrm{gh}}$ & $17.8 \pm 2.5^{\mathrm{ef}}$ & $11.9 \pm 0.7$ bc & $17.6 \pm 0.2^{\mathrm{abc}}$ & $6.0 \pm 0.4^{\mathrm{ab}}$ & $5.7 \pm 0.4^{\mathrm{bcd}}$ & $315.5 \pm 89.8^{\mathrm{abc}}$ & $835.0 \pm 198.0^{\mathrm{b}}$ & $1.02 \pm 0.3^{\mathrm{abc}}$ & $2.49 \pm 0.6^{\text {bcde }}$ & $35.42 \pm 7.5$ abcde & $45.83 \pm 8.33^{\mathrm{abcd}}$ \\
\hline TZm-1153 & $77.78 \pm 5.56^{\text {abcdefg }}$ & $12.3 \pm 0.3^{\text {efgh }}$ & $18.7 \pm 5.0^{\mathrm{def}}$ & $16.0 \pm 1.8^{\mathrm{abc}}$ & $18.2 \pm 0.9^{\mathrm{abc}}$ & $5.7 \pm 0.6^{\mathrm{abcd}}$ & $5.8 \pm 0.6^{\mathrm{bcd}}$ & $303.7 \pm 57.9^{\mathrm{bc}}$ & $1019.1 \pm 266.0^{\mathrm{ab}}$ & $0.92 \pm 0.2^{\mathrm{abc}}$ & $3.23 \pm 1.1^{\mathrm{ab}}$ & $29.17 \pm 2.1^{\text {abcdef }}$ & $47.92 \pm 2.1^{\mathrm{abcd}}$ \\
\hline $\mathrm{TZm}-1163$ & $98.15 \pm 1.85^{\mathrm{a}}$ & $11.4 \pm 1.1^{\text {fgh }}$ & $21.3 \pm 0.8^{\mathrm{def}}$ & $13.9 \pm 1.9^{\mathrm{abc}}$ & $17.1 \pm 0.5^{\mathrm{abcd}}$ & $6.2 \pm 0.4^{\mathrm{a}}$ & $5.2 \pm 0.2^{\mathrm{d}}$ & $238.4 \pm 45.0^{\text {cde }}$ & $1066.5 \pm 222.0^{\mathrm{ab}}$ & $0.81 \pm 0.2^{\mathrm{abc}}$ & $3.02 \pm 0.8^{\mathrm{abc}}$ & $43.75 \pm 9.6^{\mathrm{ab}}$ & $52.08 \pm 11.0^{\mathrm{abc}}$ \\
\hline $\mathrm{TZm}-1241$ & $67.59 \pm 1.85^{\text {defgh }}$ & $9.3 \pm 0.2^{\mathrm{gh}}$ & $17.8 \pm 2.9^{\text {ef }}$ & $12.4 \pm 0.7^{\mathrm{abc}}$ & $19.3 \pm 0.3^{\mathrm{a}}$ & $5.3 \pm 0.5^{\text {abcde }}$ & $5.2 \pm 0.1^{\mathrm{d}}$ & $202.1 \pm 41.5^{\text {cde }}$ & $944.5 \pm 21.0^{\mathrm{ab}}$ & $0.57 \pm 0.1^{\mathrm{bc}}$ & $2.61 \pm 0.4^{\mathrm{bcd}}$ & $37.50 \pm 9.6^{\mathrm{abcd}}$ & $47.92 \pm 7.5^{\text {abcd }}$ \\
\hline $\mathrm{TZm}-1427$ & $43.52 \pm 2.45^{\mathrm{ij}}$ & $8.5 \pm 0.7^{\mathrm{h}}$ & $13.7 \pm 2.1^{\mathrm{f}}$ & $13.2 \pm 0.9^{\mathrm{abc}}$ & $19.2 \pm 0.7^{\mathrm{ab}}$ & $5.2 \pm 0.5^{\text {abcdefg }}$ & $4.8 \pm 0.1^{\mathrm{d}}$ & $203.2 \pm 18.2^{\text {cde }}$ & $709.8 \pm 166.0^{\mathrm{bc}}$ & $0.55 \pm 0.1^{\mathrm{bc}}$ & $1.76 \pm 0.3^{\text {bcde }}$ & $20.83 \pm 12.7^{\text {abcdef }}$ & $33.33 \pm 12.7^{\mathrm{bcd}}$ \\
\hline TZm-299 & $87.04 \pm 3.34^{\text {abcde }}$ & $11.3 \pm 1.6^{\mathrm{fgh}}$ & $19.4 \pm 3.6^{\text {def }}$ & $14.1 \pm 0.9^{\mathrm{abc}}$ & $18.5 \pm 0.4^{\mathrm{ab}}$ & $5.8 \pm 0.6^{\mathrm{abc}}$ & $4.8 \pm 0.2^{\mathrm{d}}$ & $274.8 \pm 61.5^{\mathrm{cd}}$ & $897.6 \pm 223.0^{\mathrm{ab}}$ & $0.86 \pm 0.2^{\mathrm{abc}}$ & $2.38 \pm 0.7^{\text {bcde }}$ & $41.67 \pm 13.7^{\mathrm{abc}}$ & $58.33 \pm 14.6^{\mathrm{ab}}$ \\
\hline $\mathrm{TZm}-304$ & $62.04 \pm 6.48^{\mathrm{fghi}}$ & $11.1 \pm 0.7^{\mathrm{gh}}$ & $28.0 \pm 2.6^{\text {bcdef }}$ & $16.6 \pm 1.5^{\mathrm{ab}}$ & $18.5 \pm 1.0^{\mathrm{ab}}$ & $5.5 \pm 0.2^{\text {abcde }}$ & $6.3 \pm 0.8^{\mathrm{abcd}}$ & $505.4 \pm 68.5^{\mathrm{a}}$ & $1348.1 \pm 201^{\mathrm{a}}$ & $1.46 \pm 0.1^{\mathrm{a}}$ & $4.65 \pm 1.1^{\mathrm{a}}$ & $47.92 \pm 15.0^{\mathrm{a}}$ & $60.42 \pm 15.0^{\mathrm{ab}}$ \\
\hline $\mathrm{TZm}-378$ & $59.26 \pm 1.85^{\text {ghi }}$ & $9.5 \pm 1.1^{\mathrm{gh}}$ & $23.5 \pm 4.4^{\mathrm{def}}$ & $14.2 \pm 1.3^{\mathrm{abc}}$ & $18.9 \pm 0.7^{\mathrm{ab}}$ & $5.3 \pm 0.2^{\text {abcdef }}$ & $5.0 \pm 0.3^{\mathrm{d}}$ & $323.7 \pm 90.2^{\mathrm{abc}}$ & $1135.0 \pm 165^{\mathrm{ab}}$ & $0.95 \pm 0.3^{\mathrm{abc}}$ & $3.08 \pm 0.6^{\mathrm{abc}}$ & $31.25 \pm 12.5^{\text {abcdef }}$ & $43.75 \pm 12.5^{\mathrm{abcd}}$ \\
\hline $\mathrm{TZm}-687$ & $68.52 \pm 19.40^{\text {cdefgh }}$ & $10.2 \pm 1.6^{\mathrm{gh}}$ & $22.1 \pm 5.8^{\mathrm{def}}$ & $15.1 \pm 2.2^{\mathrm{abc}}$ & $18.8 \pm 0.5^{\mathrm{ab}}$ & $4.8 \pm 0.1^{\text {cdefg }}$ & $5.4 \pm 0.2^{\mathrm{cd}}$ & $485.0 \pm 28.2^{\mathrm{ab}}$ & $1057.2 \pm 235^{\mathrm{ab}}$ & $1.25 \pm 0.1^{\mathrm{ab}}$ & $3.17 \pm 0.9^{\mathrm{ab}}$ & $37.50 \pm 9.6^{\text {abcd }}$ & $47.92 \pm 11.6^{\mathrm{abcd}}$ \\
\hline Ikom White & $59.26 \pm 5.16^{\mathrm{ghi}}$ & $8.9 \pm 0.2^{\mathrm{h}}$ & $14.4 \pm 2.3^{\mathrm{f}}$ & $10.5 \pm 1.7^{\mathrm{bc}}$ & $16.1 \pm 0.7^{\text {abcde }}$ & $5.0 \pm 0.2^{\text {bcdefg }}$ & $4.9 \pm 0.3^{\mathrm{d}}$ & $237.5 \pm 56.3^{\text {cde }}$ & $714.9 \pm 302^{\mathrm{bc}}$ & $0.64 \pm 0.2^{b c}$ & $1.84 \pm 0.8^{\text {bcde }}$ & $14.58 \pm 7.5^{\text {bcdef }}$ & $20.83 \pm 7.5^{\mathrm{cd}}$ \\
\hline
\end{tabular}


The average number of leaves recorded for Ikom White (5.2) at 2WAS was statistically similar $(\mathrm{p}>0.05)$ to those of TZm-109 (5.5) and TZm-1241 (4.8), with the highest and lowest number of leaves per plant, respectively. Average leaf area over the two cropping seasons ranged from $183.2 \mathrm{~cm}^{2}$ to $338.6 \mathrm{~cm}^{2}$ at 2 WAS and $452.8 \mathrm{~cm}^{2}$ to $803.8 \mathrm{~cm}^{2}$ at 4 WAS. Though leaf area of accessions TZm-209 and TZm-304 were higher and significantly different $(\mathrm{p} \leq 0.05$ ) from TZm-1097, TZm-1163, TZm1241 and TZm-1427 at 2WAS, all maize accessions were not significantly different $(\mathrm{p}>0.05)$ from the check. The LAI at 2WAS was literally doubled at 4 WAS for all the maize accessions and the check. The range of LAI obtained was 0.41 to 1.07 2WAS and 1.25 to 2.88 at 4WAS. Ikom White recorded LAI of 0.64 and 1.66 at 2WAS and 4WAS, respectively. Fall armyworm (FAW) infestations were progressive and severe over the number of days after sowing within each season (Table 4) and across the seasons (Table 5). Accession TZm-304 recorded the highest FAW infestation (47.67\%) in Calabar at 2WAS. Overall FAW infestations were higher at $4 \mathrm{WAS}$ in both Calabar and Akpabuyo (Table 4). Also, though $25 \%$ of Ikom White were affected by FAW at 2WAS in Calabar, the least affected of all the maize genotypes were TZm-1163 (2.08\%), TZm-109 (4.17\%), TZm-1241 (6.25\%) and TZm-1153 (6.25\%). Across the two seasons, FAW infestations recorded for the maize accessions were not significantly different $(p>0.05)$ from the check $(19.79 \%)$ at $2 \mathrm{WAS}$, except $(\mathrm{p} \leq 0.05)$ TZm-304 (47.92\%) (Table 5). Although a study was conducted in Ghana that involved TZm-1153 and TZm1097 accessions used in the present study, one of the main aim of that study was to obtain information for the breeding potentials of the maize accession by assessing their genetic variability, diversity and relatedness using phenotypic evaluation (Twumasi et al., 2017). However, of all the 21 agro-morphological traits data obtained from Twumasi et al. (2017), no reference was made to fall armyworm damage despite the economic significance of this pest to maize production in Central and West Africa.

Genotype x season interaction (GSI) model describing early vegetative growth and fall armyworm damage on African maize accessions in early (Calabar) and late (Akpabuyo) maize cropping seasons in 2018: The precision and predictive power of a single season trial is very low and severely biased. To predict the genotypes' performance within the target population environments (TPE), the adoption of a multi-environment trial (MET) is practiced. In this study, the two maize growing seasons represented two test environments and the ten (10) African maize accessions and a local maize variety (check) represented the eleven genotypes. Early vegetative growth traits and FAW recorded higher mean values at 4WAS (Table 5). From results of the combined ANOVA, there were no significant differences $(p>0.05)$ among the genotypes for the traits studied which could imply that there were either no or nondetectable presence of genetic variability (Table 6). The non-significant $(\mathrm{p}>$ 0.05 ) mean squares for genotype confirmed that the trend of growth performance and response to FAW by these genotypes were in a similar manner in the two cropping seasons. Estimates of variance components (Table 7) showed that the proportion of genotypic variance contained in the phenotypic variance for most of the growth traits were $0 \%$, except for number of leaves per plant $(24 \%)$ at $4 \mathrm{WAS}$, stem width $(47 \%)$ and LAI $(25 \%)$ at 2 WAS and FAW (2\%) at 2 WAS. This also highlighted the fact that there was no conspicuous resistance to FAW damage among the maize accessions and the check, Ikom White, grown in Calabar and Akpabuyo. There was, however, significant genotype x season interactions (GSI) for seedling emergence $(\mathrm{p} \leq 0.001)$, plant height $(\mathrm{p} \leq$ $0.05)$, number of leaves per plant $(\mathrm{p} \leq 0.01)$, leaf area at 2 WAS $(\mathrm{p} \leq 0.01)$ and FAW damage at 4WAS $(\mathrm{p} \leq 0.05)$ (Table 6). This indicated that the maize genotypes could apparently exhibit different phenotypic performance either in the early or second cropping seasons for the five traits. These observations were inconsonance with Fentaw et al. (2015) and (Tonk et al., 2011). In considering the proportion of GSI variance in the phenotypic variance, the following traits made more than $50 \%$ contribution namely, plant height at 2 WAS $(54 \%)$, FAW damage at 4 WAS $(58 \%)$, leaf area at 2 WAS $(64 \%)$, number of leaves per plant at 2WAS (71\%) and seedling emergence $(89 \%)$ (Table 7$)$. The proportion of phenotypic variance attributed to seasonal effects was highest in leaf area (100\%) and leaf area index (92\%) at 4WAS, but slightly above $50 \%$ in FAW infestation at 2WAS. Seedling emergence was less likely to be influenced by seasonal effect (11\%). Apparently, the two experimental sites (Calabar and Akpabuyo) had few relatively similar soil properties and weather conditions. Generally low to moderate ratios of the genotypic variance to phenotypic variance $(<0.6)$ with accompanying low to moderately high genetic coefficient of variability $(\mathrm{GCV})(3.55-14.28 \%)$ indicated that the observed early vegetative growth trait(s) difference(s) in the present study were not highly heritable. There have been reports indicating that it is unreliable to consider economic yield as the only determinant for genotype performance and selection (Fentaw et al., 2015; Ittah and Obok, 2019; Kang et al., 1991; Odor et al., 2017; Tonk et al., 2011). Also, none of these reports considered the actual performance of a potential superior crop genotype at its early vegetative growth stage and initial establishments in the field as addressed in the present study. 

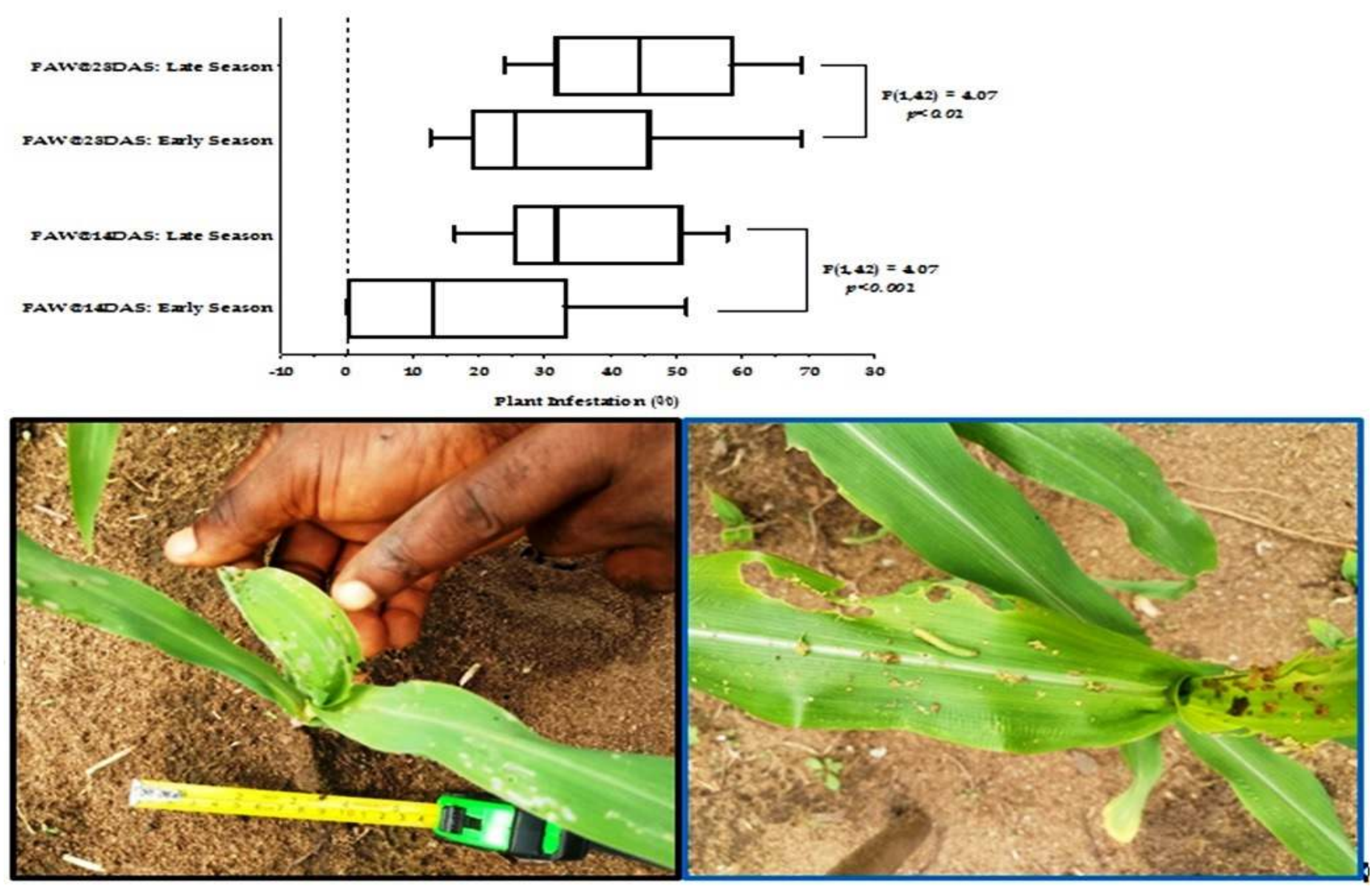

Figure 1 (top): Fall armyworm (FAW) infestation on African maize accession at 14 and 28 days after sowing (DAS) during early and second cropping seasons in 2018. Figure 2 (bottom left): FAW on a seven-day old maize seedling showing patches. Figure 3 (bottom right): FAW larval feeding with leaves defoliation; leaving moist sawdust-like frass in the funnel and on flag leaves. 
Table 5. Mean early vegetative growth performance and fall armyworm infestation on African maize accessions.

\begin{tabular}{|c|c|c|c|c|c|c|c|c|c|c|c|c|c|}
\hline \multirow[b]{2}{*}{ Genotype } & \multirow{2}{*}{$\begin{array}{l}\text { Seedling } \\
\text { Emergence (\%) }\end{array}$} & \multicolumn{2}{|c|}{ Plant Height (cm) } & \multicolumn{2}{|c|}{ Stem Width (mm) } & \multicolumn{2}{|c|}{ Number of Leaves } & \multicolumn{2}{|c|}{ Leaf Area $\left(\mathrm{cm}^{2}\right)$} & \multicolumn{2}{|c|}{ Leaf Area Index } & \multicolumn{2}{|c|}{$\begin{array}{l}\text { Fall Armyworm } \\
\text { Infestation (\%) }\end{array}$} \\
\hline & & 2WAS & 4WAS & 2WAS & 4WAS & 2WAS & 4WAS & 2WAS & 4WAS & 2WAS & 4WAS & 2WAS & 4WAS \\
\hline TZm-109 & $71.76^{\mathrm{bc}}$ & $17.1^{\mathrm{abc}}$ & $29.8^{\mathrm{ab}}$ & $13.9^{\mathrm{ab}}$ & $15.6^{\mathrm{ab}}$ & $5.5^{\mathrm{a}}$ & $6.3^{\mathrm{a}}$ & $326.1^{\mathrm{a}}$ & $625.2^{\mathrm{a}}$ & $1.07^{\mathrm{a}}$ & $2.00^{\mathrm{ab}}$ & $22.92^{\mathrm{b}}$ & $34.38^{\mathrm{b}}$ \\
\hline TZm-1097 & $61.11^{\mathrm{c}}$ & $13.9^{\mathrm{abc}}$ & $30.9^{\mathrm{ab}}$ & $11.8^{\mathrm{b}}$ & $15.6^{\mathrm{ab}}$ & $5.1^{\mathrm{ab}}$ & $6.2^{\mathrm{a}}$ & $183.2^{\mathrm{b}}$ & $492.7^{\mathrm{a}}$ & $0.81^{\mathrm{ab}}$ & $1.53^{\mathrm{ab}}$ & $22.92^{\mathrm{b}}$ & $35.42^{\mathrm{b}}$ \\
\hline TZm-1153 & $73.15^{\mathrm{bc}}$ & $16.5^{\mathrm{abc}}$ & $32.5^{\mathrm{ab}}$ & $14.5^{\mathrm{ab}}$ & $16.4^{\mathrm{ab}}$ & $5.1^{\mathrm{ab}}$ & $6.8^{\mathrm{a}}$ & $209.1^{\mathrm{ab}}$ & $594.6^{\mathrm{a}}$ & $0.68^{\mathrm{ab}}$ & $1.98^{\mathrm{ab}}$ & $17.71^{\mathrm{b}}$ & $34.38^{\mathrm{b}}$ \\
\hline TZm-1163 & $65.74^{\mathrm{bc}}$ & $12.5^{\mathrm{c}}$ & $24.5^{\mathrm{b}}$ & $12.6^{\mathrm{b}}$ & $15.0^{\mathrm{b}}$ & $5.2^{\mathrm{ab}}$ & $5.9^{\mathrm{a}}$ & $162.2^{\mathrm{b}}$ & $605.7^{a}$ & $0.57^{\mathrm{ab}}$ & $1.77^{\mathrm{ab}}$ & $22.92^{\mathrm{b}}$ & $36.46^{\mathrm{b}}$ \\
\hline $\mathrm{TZm}-1241$ & $70.83^{\mathrm{bc}}$ & $13.3^{\mathrm{bc}}$ & $26.2^{\mathrm{b}}$ & $11.2^{\mathrm{b}}$ & $15.7^{\mathrm{ab}}$ & $4.8^{\mathrm{b}}$ & $5.9^{\mathrm{a}}$ & $176.2^{\mathrm{b}}$ & $567.4^{\mathrm{a}}$ & $0.41^{\mathrm{b}}$ & $1.66^{\mathrm{ab}}$ & $21.88^{\mathrm{b}}$ & $32.29^{\mathrm{b}}$ \\
\hline $\mathrm{TZm}-1427$ & $63.43^{\mathrm{bc}}$ & $14.2^{\mathrm{abc}}$ & $25.5^{\mathrm{b}}$ & $14.3^{\mathrm{ab}}$ & $17.7^{\mathrm{ab}}$ & $5.0^{\mathrm{ab}}$ & $5.8^{\mathrm{a}}$ & $175.3^{\mathrm{b}}$ & $452.8^{\mathrm{a}}$ & $0.50^{\mathrm{ab}}$ & $1.25^{\mathrm{b}}$ & $16.67^{\mathrm{b}}$ & $33.33^{\mathrm{b}}$ \\
\hline TZm-299 & $88.89^{\mathrm{a}}$ & $18.9^{\mathrm{ab}}$ & $31.5^{\mathrm{ab}}$ & $14.7^{\mathrm{ab}}$ & $17.4^{\mathrm{ab}}$ & $5.4^{\mathrm{ab}}$ & $\begin{array}{l}5.0 \\
6.4^{\mathrm{a}}\end{array}$ & $237.2^{\mathrm{ab}}$ & $572.2^{\mathrm{a}}$ & $0.66^{\mathrm{ab}}$ & $1.71^{\mathrm{ab}}$ & $28.12^{\mathrm{ab}}$ & $44.79^{\mathrm{b}}$ \\
\hline TZm-304 & $78.24^{\mathrm{ab}}$ & $18.9^{\mathrm{ab}}$ & $39.5^{\mathrm{a}}$ & $17.5^{\mathrm{a}}$ & $18.6^{\mathrm{a}}$ & $5.5^{\mathrm{ab}}$ & $7.2^{\mathrm{a}}$ & $338.6^{\mathrm{a}}$ & $803.8^{\mathrm{a}}$ & $0.96^{\mathrm{ab}}$ & $2.88^{\mathrm{a}}$ & $47.92^{\mathrm{a}}$ & $65.62^{\mathrm{a}}$ \\
\hline $\mathrm{TZm}-378$ & $69.44^{\mathrm{bc}}$ & $13.1^{\mathrm{bc}}$ & $25.7^{\mathrm{b}}$ & $13.8^{\mathrm{ab}}$ & $16.7^{\mathrm{ab}}$ & $5.0^{\mathrm{ab}}$ & $6.3^{\mathrm{a}}$ & $206.9^{\mathrm{ab}}$ & $648.2^{\mathrm{a}}$ & $0.67^{\mathrm{ab}}$ & $1.90^{\mathrm{ab}}$ & $36.46^{\mathrm{ab}}$ & $51.04^{\mathrm{ab}}$ \\
\hline TZm-687 & $78.70^{\mathrm{ab}}$ & $15.7^{\mathrm{abc}}$ & $28.9^{\mathrm{ab}}$ & $15.3^{\mathrm{ab}}$ & $17.7^{\mathrm{ab}}$ & $4.8^{\mathrm{b}}$ & $6.5^{\mathrm{a}}$ & $284.3^{\mathrm{ab}}$ & $597.7^{\mathrm{a}}$ & $0.83^{\mathrm{ab}}$ & $1.87^{\mathrm{ab}}$ & $29.17^{\mathrm{ab}}$ & $41.67^{\mathrm{b}}$ \\
\hline Ikom White & $69.44^{b c}$ & $19.9^{a}$ & $29.1^{\mathrm{ab}}$ & $12.5^{b}$ & $16.1^{\mathrm{ab}}$ & $5.2^{\mathrm{ab}}$ & $6.5^{\mathrm{a}}$ & $252.2^{\mathrm{ab}}$ & $528.6^{\mathrm{a}}$ & $0.64^{\mathrm{ab}}$ & $1.66^{\mathrm{ab}}$ & $19.79^{b}$ & $32.29^{b}$ \\
\hline
\end{tabular}

WAS $=$ Weeks after sowing. Mean \pm standard error with the same letter(s) in superscript within the same column are not significantly different at $95 \%$ confidence limit using Duncan's Multiple Range Test.

Table 6. Mean squares of combined ANOVA of early vegetative growth and fall armyworm infestation on African maize accessions.

\begin{tabular}{|c|c|c|c|c|c|c|c|c|c|c|c|c|c|c|}
\hline \multirow[b]{2}{*}{ Source of Variation } & \multirow[b]{2}{*}{ df } & \multirow{2}{*}{$\begin{array}{l}\text { Seedling } \\
\text { Emergence (\%) }\end{array}$} & \multicolumn{2}{|c|}{ Plant Height $(\mathrm{cm})$} & \multicolumn{2}{|c|}{ Stem Width (mm) } & \multicolumn{2}{|c|}{ Number of Leaves } & \multicolumn{2}{|c|}{ Leaf Area $\left(\mathrm{cm}^{2}\right)$} & \multicolumn{2}{|c|}{ Leaf Area Index } & \multicolumn{2}{|c|}{$\begin{array}{l}\text { Fall Armyworm } \\
\text { Infestation (\%) }\end{array}$} \\
\hline & & & 2WAS & 4WAS & 2WAS & 4WAS & 2WAS & 4WAS & 2WAS & 4WAS & 2WAS & 4WAS & 2WAS & 4WAS \\
\hline Season $(\mathrm{S})^{\dagger \dagger}$ & 1 & - & - & - & - & - & - & - & - & - & - & - & - & - \\
\hline Rep (Season) $)^{\dagger \dagger}$ & 4 & - & - & - & - & - & - & - & - & - & - & - & - & - \\
\hline Genotype (G) & 10 & $369.62^{\text {ns }}$ & $41.53^{\mathrm{ns}}$ & $109.16^{\mathrm{ns}}$ & $18.963^{\mathrm{ns}}$ & $7.564^{\mathrm{ns}}$ & $0.3693^{\mathrm{ns}}$ & $0.9606^{\mathrm{ns}}$ & $22876^{\mathrm{ns}}$ & $50143^{\mathrm{ns}}$ & $0.2293^{\mathrm{ns}}$ & $1.0031^{\mathrm{ns}}$ & $506.5^{\mathrm{ns}}$ & $638.5^{\mathrm{ns}}$ \\
\hline $\mathrm{G} \times \mathrm{S}$ & 10 & $1252.53^{* * *}$ & $44.09^{*}$ & $131.56^{\mathrm{ns}}$ & $10.057^{\mathrm{ns}}$ & $7.585^{\mathrm{ns}}$ & $0.8655^{* *}$ & $0.6583^{\mathrm{ns}}$ & $29511^{* *}$ & $67007^{\mathrm{ns}}$ & $0.1542^{\mathrm{ns}}$ & $1.1302^{\mathrm{ns}}$ & $494.9^{\text {ns }}$ & $658.9^{*}$ \\
\hline Pooled error & 40 & 138.06 & 20.19 & 88.06 & 10.027 & 5.77 & 0.2725 & 0.9742 & 10514 & 71082 & 0.1873 & 1.051 & 271.7 & 275.4 \\
\hline
\end{tabular}

i† It was noted that the associated variance components for the mean squares had very large standard errors. ${ }^{-}$The degree of freedom was inadequate for the test of significance in Season (S) and Replicates within Season (Rep (Season)) to be valid. *, **, *** and ns imply $\mathrm{p} \leq 0.05, \leq 0.01, \leq 0.001$ and non-significance, respectively. 
Table 7. Estimates of variance components and their ratios for early vegetative growth and fall armyworm infestations on African maize accessions.

\begin{tabular}{|c|c|c|c|c|c|c|c|c|c|c|c|c|c|}
\hline & \multirow{2}{*}{$\begin{array}{l}\text { Seedling } \\
\text { Emergence (\%) }\end{array}$} & \multicolumn{2}{|c|}{ Plant Height (cm) } & \multicolumn{2}{|c|}{ Number of Leaves } & \multicolumn{2}{|c|}{ Stem Width (mm) } & \multicolumn{2}{|c|}{ Leaf Area $\left(\mathrm{cm}^{2}\right)$} & \multicolumn{2}{|c|}{ Leaf Area Index } & \multicolumn{2}{|c|}{$\begin{array}{l}\text { Fall Armyworm } \\
\text { Infestation }(\%)\end{array}$} \\
\hline & & 2WAS & 4WAS & 2WAS & 4WAS & 2WAS & 4WAS & 2WAS & 4WAS & 2WAS & 4WAS & 2WAS & 4WAS \\
\hline Mean & 71.9 & 15.8 & 29.5 & 5.1 & 6.3 & 13.8 & 16.6 & 231.9 & 589.9 & 0.7 & 1.8 & 26.0 & 40.2 \\
\hline$\sigma_{g}^{2}$ & $-147.15^{\dagger}$ & $-0.43^{\dagger}$ & $-3.73^{\dagger}$ & $-0.08^{\dagger}$ & 0.05 & 1.48 & $-0.01^{\dagger}$ & $-1105.79^{\dagger}$ & $-2810.61^{\dagger}$ & 0.01 & $-0.02^{\dagger}$ & 1.93 & $-3.39^{\dagger}$ \\
\hline$\sigma_{g s}^{2}$ & 371.49 & 7.97 & 14.50 & 0.20 & $-0.11^{\dagger}$ & 0.01 & 0.61 & 6332.27 & $-1358.28^{\dagger}$ & $-0.01^{\dagger}$ & 0.03 & 74.42 & 127.80 \\
\hline$\sigma_{e}^{2}$ & 138.06 & 20.19 & 88.06 & 0.27 & 0.97 & 10.03 & 5.77 & 10514.20 & 71081.60 & 0.19 & 1.05 & 271.66 & 275.45 \\
\hline$\sigma_{p h}^{2}$ & 208.75 & 7.35 & 21.93 & 0.14 & 0.21 & 3.16 & 1.26 & 4918.50 & 11846.93 & 0.04 & 0.19 & 84.42 & 109.81 \\
\hline$\sigma_{g}^{2} / \sigma_{p h}^{2}$ & - & - & - & - & 0.24 & 0.47 & - & - & - & 0.25 & - & 0.02 & - \\
\hline$\left[\frac{\sigma_{g s}^{2}}{s}\right] / \sigma_{p h}^{2}$ & 0.89 & 0.54 & 0.33 & 0.71 & - & 0.002 & 0.24 & 0.64 & - & - & 0.08 & 0.44 & 0.58 \\
\hline$\left[\frac{\sigma_{e}^{2}}{s r}\right] / \sigma_{p h}^{2}$ & 0.11 & 0.46 & 0.67 & 0.32 & 0.77 & 0.53 & 0.76 & 0.36 & 1.00 & 0.79 & 0.92 & 0.54 & 0.42 \\
\hline GCV (\%) & - & - & - & - & 3.55 & 8.82 & - & - & - & 14.28 & - & 5.34 & - \\
\hline PCV (\%) & 20.09 & 17.16 & 15.87 & 7.34 & 7.27 & 12.88 & 6.76 & 30.24 & 18.45 & 28.57 & 24.22 & 35.33 & 26.07 \\
\hline
\end{tabular}

†Value is negative, estimated as zero and represented as ' '- in subsequent calculations. $\mathrm{s}=$ season, $\mathrm{g}=$ genotype, $\mathrm{r}=$ replicate $\sigma_{g}^{2}=$ genotypic variance, $\sigma_{s}^{2}=$ season variance, $\sigma_{g s}^{2}=$ genotype x season variance, $\sigma_{r(s)}^{2}=$ replicate within season variance, $\sigma_{e}^{2}=$ pooled error variance, $\sigma_{p h}^{2}=\sigma_{g}^{2}+\frac{\sigma_{g s}^{2}}{s}+\frac{\sigma_{e}^{2}}{s r}=$ phenotypic variance, GCV $=$ genotypic coefficient of variability and PCV = phenotypic coefficient of variability. 
Conclusions: The plant breeder is often faced with the task of exploiting genetic diversity for the improvement of a crop's economic yield and yield-related traits. This could be by the modification of abiotic factors especially growing condition and requirements e.g. soil fertility and irrigation. However, it is not uncommon to unintentionally ignore biotic factors such as an invasive pest. It is always safe to consider those traits in a crop that could give information on their early growth and performance when challenged with biotic stresses. These traits and their timing could serve as aids to selection for maize accessions that could serve as possible breeding materials for improvement of maize against fall armyworm during early vegetative growth stages, field establishments and subsequently, avoid yield losses. Our results showed that fall armyworm infestation was higher in the second planting season than in the first planting season. However, the GSI model used in the current study could be improved to shed lights on the number of years or locations at which trials should be conducted. To achieve this improvement, a genotype $\mathrm{x}$ locations $\mathrm{x}$ year (GLY) model could be adopted in subsequent multienvironment trial (MET) studies to permit well-informed decisions on number of years, locations (sites) and replicates essential to attain a robust level of precision in the assessment of the response of African maize genotypes to fall armyworm (FAW) damage during the early vegetative growth stages.

\section{REFERENCES}

Akpan-Idiok, A. U. (2012). Physicochemical properties, degradation rate and vulnerability potential of soils formed on coastal plain sands in Southeast, Nigeria. Int. J. of Agric. Res. 7: 358-366. doi: 10.3923/ijar.2012.358.366

Bates, R.G. (1973). Determination of $\mathrm{pH}$ : theory and practice. New York: Wiley.

Baudron, F., M.A. Zaman-Allah, I. Chaipa, N. Chari and P. Chinwada (2019). Understanding the factors influencing fall armyworm (Spodoptera frugiperda J.E. Smith) damage in African smallholder maize fields and quantifying its impact on yield. A case study in Eastern Zimbabwe. Crop Prot. 120: 141-150. doi: 10.1016/j.cropro.2019.01.028

Black, C.A., D.D. Evans, J.L. White, L.E. Ensminger and F.E. Clark (1965). Methods of soil analysis. In: Methods of soil analysis, Agronomy, 1572. Madison, Wis.: American Society of Agronomy.

Brévault, T., A. Ndiaye, D. Badiane, A.B. Bal, M. Sembène, P. Silvie and J. Haran (2018). First records of the fall armyworm, Spodoptera frugiperda (Lepidoptera: Noctuidae), in Senegal. Entomol Gen. 37: 129-142. doi: 10.1127/entomologia/2018/0553
Burtet, L. M., O. Bernardi, A. A. Melo, M.P. Pes, T.T. Strahl and J.V.C. Guedes (2017). Managing fall armyworm, Spodoptera frugiperda (Lepidoptera: Noctuidae), with $B t$ maize and insecticides in southern Brazil. Pest Manag. Sci. 73: 2569-2577. doi: 10.1002/ps.4660

Can, N.D. and T. Yoshida (1999). Genotypic and phenotypic variances and covariances in early maturing grain sorghum in a double cropping. Plant Prod. Sci. 2: 67-70. doi: 10.1626/pps.2.67

Chen, M., R.N. Nagoshi, I. Dhanani, R. Asokan, H.M. Mahadevaswamy, C.M. Kalleshwaraswamy, S and R. L. Meagher (2019). Genetic characterization of fall armyworm infesting South Africa and India indicate recent introduction from a common source population. PLoS One 14: e0217755. doi: 10.1371/journal.pone.0217755

Eijnatten, C.L.M. van (1965). Towards the improvement of maize in Nigeria, $\mathrm{PhD}$ Thesis, Agricultural University, Wageningen, The Netherlands.

Elings, Anne (2000). Estimation of leaf area in tropical maize. Agron. J. 92: 436-444. doi: 10.2134/agronj2000.923436x

Falconer, D.S. (1960). Introduction to quantitative genetics: Oliver \& Boyd, Edinburgh \& London.

Fentaw, A., E. Melkamu and M. Yeshitila (2015). Genotype-environment interaction and stability analysis of hybrid maize varieties in North West Ethiopia. Int. J. of Plant Breed. and Genet. 9: 247-254.

Food and Agriculture Organization of the United Nations (2018). Integrated management of the fall armyworm on maize: a guide for farmer field schools in Africa. Rome, Italy: FAO.

Goergen, G., P. L. Kumar, S. B. Sankung, A. Togola and M. Tamo (2016). First report of outbreaks of the fall armyworm Spodoptera frugiperda (J.E. Smith) (Lepidoptera, Noctuidae), a new alien invasive pest in West and Central Africa. PLoS One 11: e0165632. doi: 10.1371/journal.pone. 0165632

Gomez, K. A. and A. A. Gomez (1984). Statistical procedures for agricultural research. Singapore: Wiley-Interscience Publication, John Wiley and Sons, Inc.

Hay-Roe, M.M., R.L. Meagher, R.N. Nagoshi and Y. Newman (2016). Distributional patterns of fall armyworm parasitoids in a corn field and a pasture field in Florida. Bio. Control 96: 48-56. doi:

https://doi.org/10.1016/j.biocontrol.2016.02.003

Ittah, M.A. and E.E. Obok (2019). Breeding potential and multivariate analyses of morphological and yield traits in industrial sugarcane (Saccharum 
officinarum L.) accessions in a humid tropical agroecology. Int. J. of Plant \& Soil Sci. 27: 1-10.

Kalleshwaraswamy, C. M., R. Asokan, H. M. Mahadevaswamy and Sharanabasappa (2019). First record of invasive fall armyworm, Spodoptera frugiperda (J.E. Smith) (Lepidoptera: Noctuidae) on rice (Oryza sativa). India. J. of Entomol. and Zool. Stud. 7: 332-337.

Kang, M.S., D.P. Gorman and H.N. Pham (1991). Application of a stability statistic to international maize yield trials. Theor. Appl. Genet. 81: 162165. doi: $10.1007 / \mathrm{BF} 00215718$

Kehr, W. R. and C. O. Gardner (1960). Genetic variability in Ranger alfalfa. Agron. J. 52: 4144.

Klute, Arnold (1986). Methods of soil analysis Part 1: Physical and mineral methods. In: Methods of soil analysis Part 1: Physical and mineral methods, ed. Arnold Klute, Agronomy, 770. Madison, Wis.: American Society of Agronomy : Crop Science Society of America : Soil Science Society of America.

Marenco, R. J., R.E. Foster and C.A. Sanchez (1992). Sweet Corn Response to Fall Armyworm (Lepidoptera: Noctuidae) Damage During Vegetative Growth. J. of Econ. Entomol. 85: 1285-1292. doi: 10.1093/jee/85.4.1285

Meagher, R. L., G.S. Nuessly, R.N. Nagoshi and M.M. Hay-Roe (2016). Parasitoids attacking fall armyworm (Lepidoptera: Noctuidae) in sweet corn habitats. Bio. Control 95: 66-72. doi: https://doi.org/10.1016/j.biocontrol.2016.01.006

Murphy, J. and J. P. Riley (1962). A modified single solution method for the determination of phosphate in natural waters. Anal. Chim. Acta. 27: 31-36. doi: https://doi.org/10.1016/S00032670(00)88444-5

Nagoshi, R. N., D. Koffi, K. Agboka, K. A. Tounou, R. Banerjee, J. L. Jurat-Fuentes and R. L. Meagher (2017). Comparative molecular analyses of invasive fall armyworm in Togo reveal strong similarities to populations from the eastern United States and the Greater Antilles. PLoS One 12: e0181982. doi: 10.1371/journal.pone.0181982

Nagoshi, R. N., G. Goergen, K.A. Tounou, K. Agboka, D. Koffi and R.L. Meagher (2018). Analysis of strain distribution, migratory potential, and invasion history of fall armyworm populations in northern Sub-Saharan Africa. Sci. Rep. 8: 3710. doi: 10.1038/s41598-018-21954-1

Nigerian Meteorological Agency (NiMET) (2018). Agrometeorological Bulletin. In: Agrometeorological Bulletin. Bill Clinton Drive, Nnamdi Azikiwe International Airport, Abuja:
National Weather Forecasting and Climate Research Centre.

Odor, E. O., G. A. Iwo and E. E. Obok (2017). Graphical assessment of yield stability and adaptation of cucumber (Cucumis sativus L) genotypes in Cross River State, Nigeria. J. of Agric. and Crop Res. 5: 108-116.

Oliveira, M. A., F. H. I. Filho and R.T. Thuler (2018). Eficiency of Bacillus thuringiensis in controlling the corn fall armyworm in laboratory conditions. Revista Inova Ciência \& Tecnologia, Uberaba 4: 14-19.

Onwudike, S. U. (2015). Effect of land use types on vulnerability potential and degradation rate of soils of similar lithology in a tropical soil of Owerri, Southeastern Nigeria. Int. J. of Soil Sci. 10: 177-185. doi: 10.3923/ijss.2015.177.185

Otim, M. H., W. T. Tay, T. K. Walsh, D. Kanyesigye, S. Adumo, J. Abongosi, S. Ochen, J. Sserumaga, S. Alibu, G. Abalo, G. Asea and A. Agona (2018). Detection of sister-species in invasive populations of the fall armyworm Spodoptera frugiperda (Lepidoptera: Noctuidae) from Uganda. PLoS One 13: e0194571. doi: 10.1371/journal.pone.0194571

Pannuti, L. E., E. L. Baldin, T. E. Hunt and S. V. PaulaMoraes (2016). On-plant larval movement and feeding behavior of fall armyworm (Lepidoptera: Noctuidae) on Reproductive Corn Stages. Environ. Entomol 45: 192-200. doi: 10.1093/ee/nvv159

Prasanna, B.M., J. E. Huesing, R.Eddy and V. M. Peschke (2018). Fall armyworm in Africa: A Guide for integrated pest management, (1st ed.) eds. B.M. Prasanna, J. E. Huesing, R. Eddy and V.M. Peschke. Mexico: CDMX: CIMMYT.

Rwomushana, I., M. Bateman, T. Beale, P. Beseh, K. Cameron, M. Chiluba, V. Clottey, T. Davis, R. Day, R. Early, J. Godwin, P. Gonzalez-Moreno, M. Kansiime, M. Kenis, F. Makale, I. Mugambi, S. Murphy, Nunda. W., N. Phiri, C. Pratt and J Tambo (2018). Fall armyworm: impacts and implications for Africa. Evidence Note: Knowledge for Life. United Kingdom: Oxfordshire: CABI.

Sparks, D. L., A. L. Page, P. A. Helmke and R. H. Loeppert (1996). Methods of Soil Analysis Part 3-Chemical Methods. In Methods of Soil Analysis Part 3-Chemical Methods, eds. D. L. Sparks, A. L. Page, P. A. Helmke and R. H. Loeppert, SSSA Book Series, 1390. Madison, WI: Soil Science Society of America, American Society of Agronomy.

Toepfer, S., U. Kuhlmann, M. Kansiime, D. O. Onyango, T. Davis, K. Cameron and R. Day (2018). Communication, information sharing, and 
advisory services to raise awareness for fall armyworm detection and area-wide management by farmers. J. of Plant Diseases and Prot. 126: 103-106. doi: 10.1007/s41348-018-0202-4

Tonk, F. A., E. Ilker and M. Tosun (2011). Evaluation of genotype $\mathrm{x}$ environment interactions in maize hybrids using GGE biplot analysis. Crop Breed. and Appl. Biotech. 11: 01-09.

Twumasi, P., A. Y Tetteh, K. B. Adade, S. Asare and R. A. Akromah (2017). Morphological diversity and relationships among the IPGRI maize (Zea mays L) landraces held in IITA. Maydica 62:M25.

WorldWeatherOnline (2018). Monthly Climate Averages: Calabar and Akpabuyo - Cross River State, Nigeria. In Monthly Climate Averages: Calabar and Akpabuyo - Cross River State, Nigeria.

Yassin, T. E. (2009). Genotypic and phenotypic variances and correlations in field beans (Vicia faba L.). The J. of Agric. Sci. 81: 445-448. doi: $10.1017 / \mathrm{S} 0021859600086494$. 